\title{
Immunomodulatory properties of Tamm-Horsfall glycoprotein (THP) and uromodulin
}

\begin{abstract}
Background: Tamm-Horsfall glycoprotein (THP) and uromodulin are the most abundant glycoproteins in non-pregnant womenôs/menôs and pregnant womenôs urine, respectively. However, the bioactivities of these glycoproteins are still unclear.

Objective: To evaluate the immunomodulatory properties of THP and uromodulin on human peripheral blood mononuclear cells (PBMC). Methods: THP and uromodulin isolated with diatomaceous earth filtration were subjected to several bioassays, such as MTS viability assay, immunophenotyping and cytokine analysis.

Results: MTS viability assay and immunophenotyping analysis showed that uromodulin has greater inhibitory activities in suppressing PBMC viability and the percentage of CD4+ T helper cells and CD8+ cytotoxic T cells, compared to that of THP. In cytokine analysis, THP tended to induce pro-inflammatory cytokines such as IL-16, TNF and Th1 cytokine IFN-J; while uromodulin only induced IL-16 and suppressed both Th1 cytokine IFN-כ and Th2 cytokine IL-10.

Conclusion: These results demonstrate that uromodulin has greater immunosuppressive activities and lower inductive property in relation to activation of immune cells, which provides a more tolerant environment for the developing fetus.
\end{abstract}

Keyword: Cytokine; Immunophenotyping; Immunosuppressive; MTS assay; Tamm-Horsfall glycoprotein; Uromodulin 